\title{
Excitatory-inhibitory balance within EEG microstates and resting-state fMRI networks: assessed via simultaneous trimodal PET-MR-EEG imaging
}

Ravichandran Rajkumar (10) ${ }^{1,2,3}$, Cláudia Régio Brambilla (iD ${ }^{1,2,3}$, Tanja Veselinović $\mathbb{D}^{2}$, Joshua Bierbrier ${ }^{1,4}$,

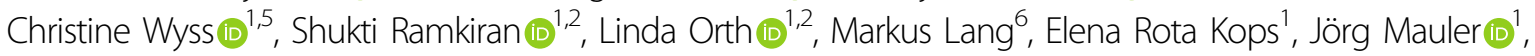
Jürgen Scheins ${ }^{1}$, Bernd Neumaier $\mathbb{1 0}^{6}$, Johannes Ermert ${ }^{6}{ }^{6}$, Hans Herzog ${ }^{1}$, Karl-Josef Langen ${ }^{1,3,7}$, Ferdinand Christoph Binkofski ${ }^{1,3,8}$, Christoph Lerche $\mathbb{D}^{1}$, N. Jon Shah ${ }^{1,3,8,9}$ and Irene Neuner $\mathbb{D}^{1,2,3}$

\begin{abstract}
The symbiosis of neuronal activities and glucose energy metabolism is reflected in the generation of functional magnetic resonance imaging (fMRI) and electroencephalography (EEG) signals. However, their association with the balance between neuronal excitation and inhibition (E/I-B), which is closely related to the activities of glutamate and $\gamma$ aminobutyric acid (GABA) and the receptor availability (RA) of GABA $A$ and mGluR5, remains unexplored. This research investigates these associations during the resting state (RS) condition using simultaneously recorded PET/MR/EEG (trimodal) data. The trimodal data were acquired from three studies using different radio-tracers such as, $\left[{ }^{11} \mathrm{C}\right] \mathrm{ABP} 688$ (ABP) $\left.(N=9),{ }^{11} \mathrm{C}\right]$ Flumazenil (FMZ) $(N=10)$ and $2-\left[{ }^{18} \mathrm{~F}\right]$ fluoro-2-deoxy-D-glucose (FDG) $(N=10)$ targeted to study the mGluR5, GABA $A_{A}$ receptors and glucose metabolism respectively. Glucose metabolism and neuroreceptor binding availability (non-displaceable binding potential $\left(\mathrm{BP}_{\mathrm{ND}}\right)$ ) of $\mathrm{GABA}_{\mathrm{A}}$ and $\mathrm{mGluR5}$ were found to be significantly higher and closely linked within core resting-state networks (RSNs). The neuronal generators of EEG microstates and the fMRI measures were most tightly associated with the $\mathrm{BP}_{\mathrm{ND}}$ of $\mathrm{GABA}_{\mathrm{A}}$ relative to mGluR5 $\mathrm{BP}_{\mathrm{ND}}$ and the glucose metabolism, emphasising a predominance of inhibitory processes within in the core RSNs at rest. Changes in the neuroreceptors leading to an altered coupling with glucose metabolism may render the RSNs vulnerable to psychiatric conditions. The paradigm employed here will likely help identify the precise neurobiological mechanisms behind these alterations in fMRI functional connectivity and EEG oscillations, potentially benefitting individualised healthcare treatment measures.
\end{abstract}

\section{Introduction}

It is generally acknowledged that the functional architecture of the brain is governed by fundamental principles such as functional segregation and integration ${ }^{1}$.

\footnotetext{
Correspondence: Irene Neuner (i.neuner@fz-juelich.de)

${ }^{1}$ Institute of Neuroscience and Medicine 4, INM-4, Forschungszentrum Jülich, Jülich, Germany

2Department of Psychiatry, Psychotherapy and Psychosomatics, RWTH Aachen University, Aachen, Germany

Full list of author information is available at the end of the article

These authors contributed equally: C. Régio Brambilla, Tanja Veselinović
}

Functional integration (both intrinsic and induced) in the brain is directed by the balance between neuronal excitation and inhibition and is closely related to activity in the brain's main excitatory and inhibitory neurotransmitters, glutamate and $\gamma$-aminobutyric acid (GABA), respectively ${ }^{2}$. This excitatory/inhibitory balance (E/I-B) has been shown to play an important role in several crucial mental processes ${ }^{3}$. It is postulated that this balance is the underlying mechanism influencing both intrinsic and stimulus-induced activity across brain regions and

\section{(c) The Author(s) 2021}

(c) (i) Open Access This article is licensed under a Creative Commons Attribution 4.0 International License, which permits use, sharing, adaptation, distribution and reproduction cc) in any medium or format, as long as you give appropriate credit to the original author(s) and the source, provide a link to the Creative Commons license, and indicate if changes were made. The images or other third party material in this article are included in the article's Creative Commons license, unless indicated otherwise in a credit line to the material. If material is not included in the article's Creative Commons license and your intended use is not permitted by statutory regulation or exceeds the permitted use, you will need to obtain permission directly from the copyright holder. To view a copy of this license, visit http://creativecommons.org/licenses/by/4.0/. 
may also play a role in the constitution of the higher cognitive functions, such as self and consciousness ${ }^{2}$. Conversely, disturbances in the E/I-B may lead to functional network alterations and have been shown to provide the underlying pathological framework in some psychiatric diseases ${ }^{4}$. Thus, a profound understanding of the neurobiological basis of the E/I-B remains a major scientific challenge and a deeper comprehension of it would offer greater insight into psychiatric diseases.

The E/I-B has been extensively investigated at the cellular level of neural activity but remains less well described at the more complex levels where it becomes interspersed with various aspects of brain function. Details relating to the mechanistic aspects of the E/I-B are assessable using different neuroimaging methods, with each method providing information pertaining to a somewhat different aspect of neural activity. Functional magnetic resonance imaging (fMRI) records the haemodynamic response-the blood oxygenation leveldependent (BOLD) contrast-which is likely to be driven by balanced proportional changes in the excitation and inhibition of neurons ${ }^{5}$. Multichannel electroencephalography (EEG) reflects voltage changes resulting from the synchronous firing of groups of neurons in the brain and thus mainly exposes the neuroelectric activity at the synapse. Positron emission tomography (PET) uses specific radioligands to visualise and quantify a variety of metabolic and physiological processes in vivo by shortliving positron-emitting radioisotopes (like ${ }^{11} \mathrm{C},{ }^{18} \mathrm{~F}$ ) used to label biological molecules or drugs $^{6}$. One of the metabolic parameters derived from PET imaging includes non-displaceable binding potential $\left(\mathrm{BP}_{\mathrm{ND}}\right)^{7}$, which specifically indicates the receptor availability (RA) depending on the used tracer.

Alone, each of these techniques provides valuable insight into the examined processes in terms of its own frame of reference. However, each technique is also constrained by its own limitations. In recent years, the development of a combined approach-simultaneous trimodal PET-MR-EEG imaging ${ }^{8}$-has enabled the measurement of different aspects of the same process as it occurs, under the same physiological and psychological conditions, shedding valuable light on the relationship between them. Thus, this simultaneous trimodal approach is suitable to elucidate the coupling between neuronal activities, energy consumption, oscillations and the E/I-B.

To date, our investigations using simultaneous trimodal MR-PET-EEG imaging have mainly focused on the default mode network (DMN), as this is the first described and one of the most prominent resting-state networks (RSNs), containing brain regions critical for several cognitive functions. Using the trimodal approach, it was possible to demonstrate a significantly higher glucose metabolism (quantified by a $2-\left[{ }^{18} \mathrm{~F}\right]$ fluoro-2-deoxy-D-glucose PET (FDG-PET)) in the DMN compared to the whole-brain non-DMN grey matter (GM) during resting state $(\mathrm{RS})^{8}$. Further, the neuronal activation within the DMN (as assessed with fMRI) was positively correlated with the metabolic activity (assessed as the mean standard uptake value of FDG). Electrical source localisation of EEG signals also showed a unique frequency range pattern.

In another investigation, a more detailed clarification of the associations between the EEG signals and the ongoing glucose energy metabolism during rest was sought by specifically focussing on EEG microstates ${ }^{9}$. The spatial distribution of active neuronal sites that contribute to electrical activity in the brain can be revealed using EEG scalp topographies. Such topographies are found to be quasi-stable for periods of about $80-120 \mathrm{~ms}^{10}$. RS EEG studies have consistently identified four topographies (usually labelled as A-D) ${ }^{11}$, and each of these topographies is named as a "microstate map"11,12. The orientation of the four microstate maps are right-frontal leftposterior (A), left-frontal right-posterior (B), midline frontal-occipital $(C)$, and midline frontal (D). The functional interpretation of microstate map assumes that each map (topography) is generated by diverse neuronal assemblies. Their locations can be estimated using source localisation techniques, such as the standard lowresolution brain electrotomography (sLORETA) ${ }^{13}$. The transition of microstate maps may be regarded as a sequential activation of various neuronal assemblies. Van De Ville et al. have indicated the microstates as being "atoms of thought" (the shortest constituting elements of cognition $)^{14}$. A small number of studies have investigated the relationship between EEG microstates and RS-fMRI using simultaneously recorded RS-EEG and fMRI, and have highlighted the four typical microstates likely to represent the RSNs usually identified in RS-fMRI studies ${ }^{14-16}$. With our own trimodal approach, we demonstrated previously tight associations between fMRI metrics, FDG-PET standard uptake values and the single microstates, indicating a functional relationship between cortical hubs, connectivity parameters and the glucose metabolism ${ }^{9}$.

However, the understanding of these complex interconnections in the context of the E/I-B is very sparse. As the EEG microstates from large-scale neural networks are a result of synaptic electrical activity due to the excitatory and inhibitory actions of the neurotransmitters, we hypothesise that the neuronal generators of EEG microstates are tightly associated not only with the functional connectivity measures and glucose metabolism but also with the availability of the neuroreceptors relevant for the E/I-B. Thus, we have chosen the following two receptors as representatives of the E/I-B for our investigation: the 
excitatory G-protein-coupled metabotropic glutamate receptor type 5 (mGluR5) and the inhibitory, ligand-gated, ion-specific $\mathrm{GABA}_{\mathrm{A}}$ receptor. For their quantification within the PET part of the trimodal MR-PET-EEG imaging, two selective radioligands were used to determine the RA: $\left[{ }^{11} \mathrm{C}\right] \mathrm{ABP} 688$ (ABP) for $\mathrm{mGluR} 55^{17}$ and $\left[{ }^{11} \mathrm{C}\right]$-Flumazenil (FMZ) for $\mathrm{GABA}_{\mathrm{A}}$ receptors ${ }^{18}$. Further, 2- $\left[{ }^{18} \mathrm{~F}\right]$-fluoro-2-desoxy-D-glucose (FDG) was used to study glucose metabolism. During each of these three separate investigations, fMRI and EEG data were assessed simultaneously with identical protocols. The data obtained were analysed jointly in an explorative approach to address the following questions:

1. To what extent does the E/I-B (expressed as the RA of mGluR5 and $G_{A B A}$ ) and the glucose metabolism measured via FDG-PET determine the activity of the neuroelectric generators of microstates as estimated by sLORETA during RS?

2. Is the previously shown higher metabolic activity within the DMN during the RS associated with a higher RA of the mGluR5 and $\mathrm{GABA}_{\mathrm{A}}$ as an expression of the higher energy demand to maintain the E/I-B in the RS?

3. In view of the tight association of the neuronal activity (regardless of whether excitatory and inhibitory) with the glucose metabolism, is there a link between the RA of the mGluR5 and $\mathrm{GABA}_{\mathrm{A}}$ and the glucose metabolism measured via FDG-PET?

4. In light of the known modulatory effect of the excitatory and inhibitory neurotransmission on the BOLD signal ${ }^{19,20}$, is there an association between the RS functional connectivity and the RA of mGluR5 and GABAA within the RSNs?

RS-fMRI data can be analysed using several methods and the possibility to derive various RS-fMRI connectivity measures has also been reported ${ }^{21}$. In this study, the RSfMRI measures such as regional homogeneity $(\mathrm{ReHo})^{22}$, degree centrality $(\mathrm{DC})^{23}$ and fractional amplitude of lowfrequency fluctuations (fALFF) $)^{24}$ are considered for finding association with neuroelectric generators of microstates and RA of mGluR5 and GABA . The chosen RSfMRI measures are distinct, data driven and do not require prior definition of seed voxels/regions. The ReHo measure considers only neighbouring voxels, thus it depicts short-range functional connectivity. The DC measure considers all voxels, so it is considered a longrange functional connectivity measure. The fALFF measure characterises slow fluctuations of the BOLD signal.

\section{Results}

The simultaneous trimodal data acquisition was successfully completed in 29 subjects. The PET data were reconstructed and parametric images representing $\mathrm{BP}_{\mathrm{ND}}$ and SUV were calculated as described in the "Methods"

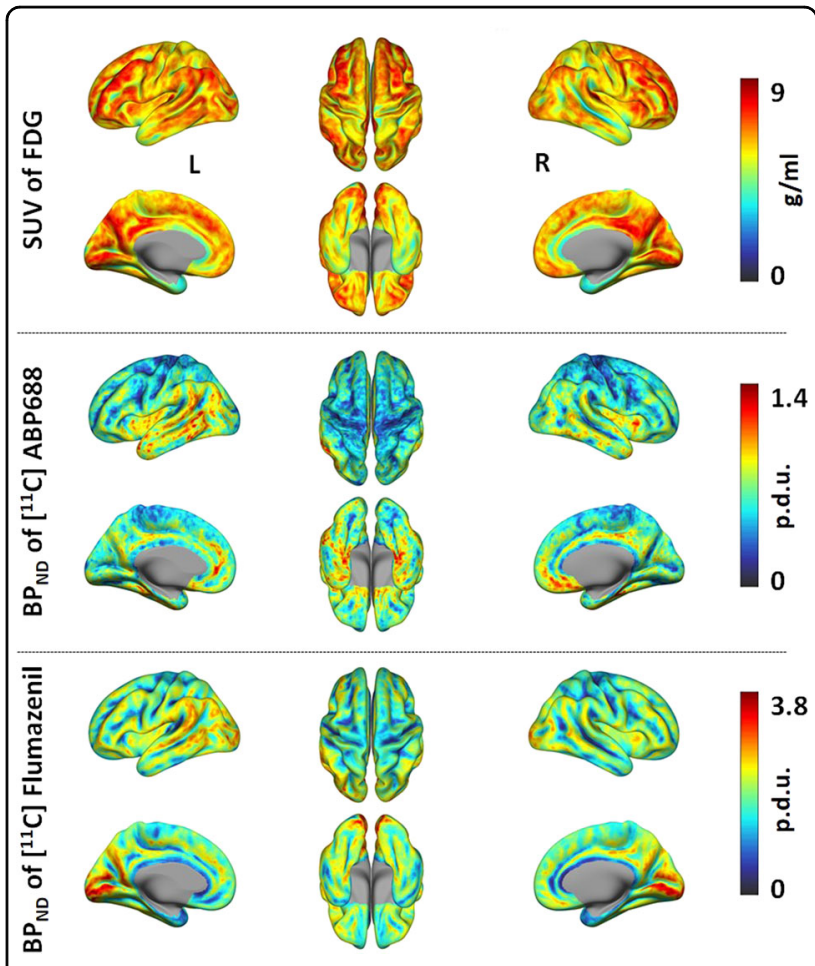

Fig. 1 PET parametric images. The 3D images representing the SUV of the FDG tracer are shown in the top row; the $\mathrm{BP}_{\mathrm{ND}}$ of $\mathrm{ABP}$ and FMZ are shown in the middle and bottom rows. The SUV images show glucose consumption in $\mathrm{g} / \mathrm{ml}$, the $\mathrm{BP}_{\mathrm{ND}}$ images show the distribution of the neuroreceptor availabilities for mGluR5 (middle row) and $\mathrm{GABA}_{\mathrm{A}}$ (bottom row) receptors in procedure defined unit (p.d.u.). The calculated PET parametric images, averaged across all subjects in each study, are presented. The left, superior and right views are shown in the upper rows and left medial, inferior and right medial views are shown in the lower rows of each parametric images.

section. The calculated PET measures are shown in Fig. 1. The artefacts in the EEG data were removed and the microstates were calculated. The three-dimensional (3D) cortical current density distribution for each microstate was calculated using the sLORETA method. The calculated microstates of an exemplary subject and the constructed 3D brain network masks for each microstate are shown in Fig. 2. Similarly, after pre-processing of the fMRI data, the RS-fMRI measures (ReHo, DC and fALFF) were calculated for each subject as described in the "Methods" section. The calculated RS-fMRI measures averaged across all the 29 subjects are shown in Fig. 3.

\section{Association between RA and EEG microstates}

The Pearson linear correlation coefficients computed between the PET and source- localised microstate measures showed significant, low positive correlations $(p<$ 0.01) with $\mathrm{GABA}_{\mathrm{A}}$ and mGluR5 (Fig. 4), remaining significant after corrections for multiple comparison. The 


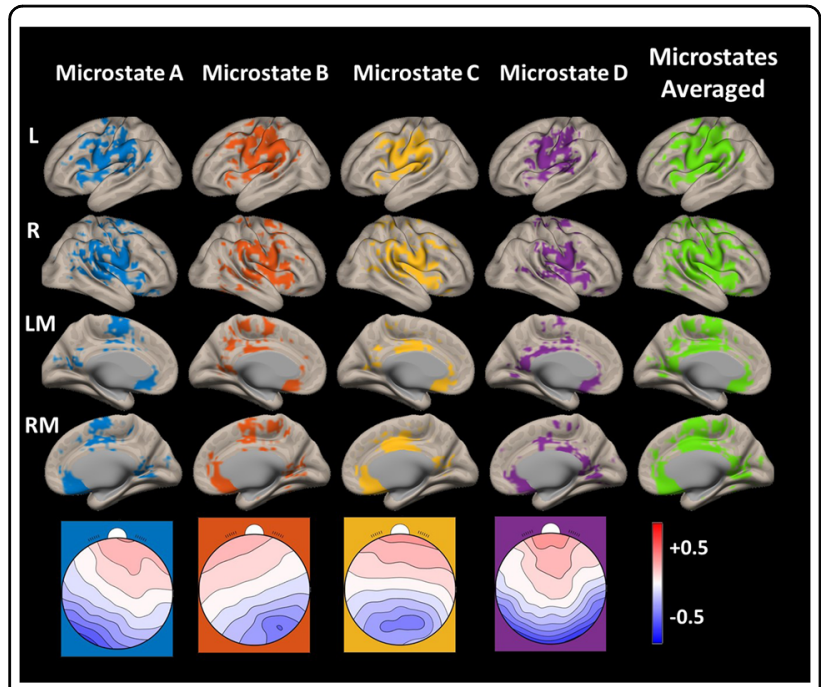

Fig. 2 EEG microstates. The topographic maps of the EEG microstates an exemplary subject (bottom row) and correspondingbrain networks masks of each microstate identified using topographic electrophysiological state source-imaging (TESS) method. The coloured regions in the 3D brain networks masks depict the regions of neuronal generators of EEG microstates. The last column images show the average image of the microstates brain network masks. The left $(\mathrm{L})$, right $(\mathrm{R})$, left medial $(\mathrm{LM})$ and right medial (RM) views are shown in each row of the 3D source-localised images.

correlation coefficients $(r)$ are summarised in Supplementary Table 1.

\section{Receptor availabilities}

The Dunn's test showed that the RA of $\mathrm{GABA}_{\mathrm{A}}$ and mGluR5 in the core RSNs were significantly $(p<0.05)$ higher compared to GM or SMN (Fig. 5) for the withinPET measure analysis.

\section{Association between RA and glucose metabolism}

The one-sample Kolmogorov-Smirnov test showed the extracted voxel values from the averaged PET, fMRI, and EEG measures to follow a normal distribution in all cases. The Pearson linear correlation coefficients $(r)$ computed between the $\mathrm{BP}_{\mathrm{ND}}$ values and FDG-PET SUV showed significant positive correlations $(p<0.01)$ within $\mathrm{GM}$ as well as the core RSN regions. The correlation results after family-wise error correction are summarised in the bar plot (Fig. 6).

\section{Association between RA and RS-fMRI measures}

The Pearson linear correlation coefficients computed between the PET and fMRI measures showed significant positive correlations $(p<0.01)$ within GM as well as the core RSNs (Fig. 7) with corrections for FWER. The correlation coefficients $(r)$ are summarised in Supplementary Table 2.

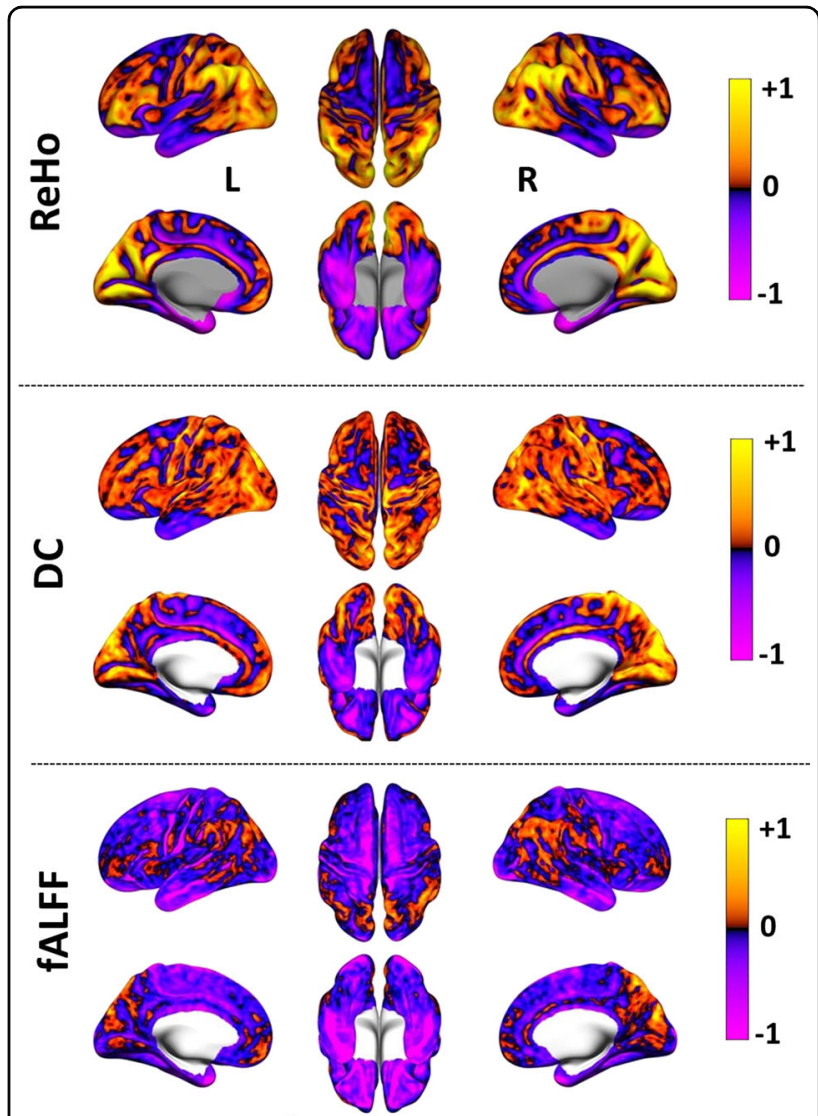

Fig. 3 RS-fMRI measures. Z-value standardised measures 3D images representing regional homogeneity (ReHo-top row), degree centrality (DC-middle row) and fractional amplitude of lowfrequency fluctuations (fALFF-bottom row) RS-fMRI measures averaged across all subjects are shown. The positively coloured regions in the $3 \mathrm{D}$ images show higher short-range functional connectivity in ReHo, long-range functional connectivity in DC and BOLD signal fluctuations in fALFF. The left, superior and right views are shown in the upper rows and left medial, inferior and right medial views are shown in the lower rows of each RS-fMRI measure.

\section{Discussion}

The E/I-B is crucial for the maintenance of the brain's functional integration. In this study, we examined the neurobiological correlates involved in E/I-B within resting-state fMRI networks using simultaneously recorded PET-MR-EEG trimodal imaging data. This unique technology enabled elucidation of the coupling between neuronal activities, energy consumption, neuronal generators of EEG microstates and the availability of the mGluR5 and the $\mathrm{GABA}_{\mathrm{A}}$ neuroreceptors-fundamental for excitatory and inhibitory neurotransmission. Our study demonstrates the feasibility and advantages of measuring trimodal data simultaneously using different radioligands and illustrates at the same time a new approach to studying associations between neuronal activities through various measures derived from 


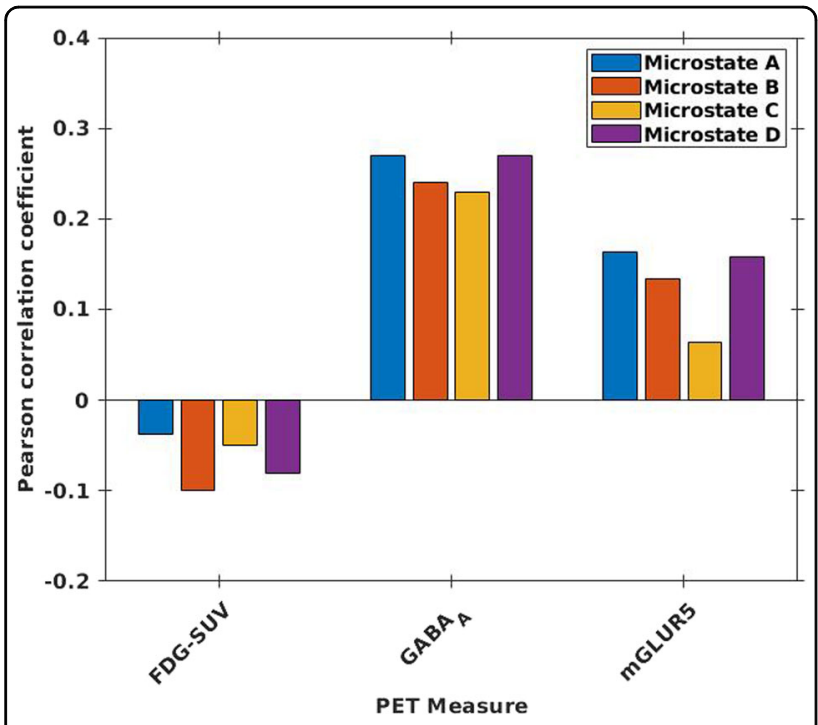

Fig. 4 Correlation values between PET and microstates. The correlation values $(p<0.01)$ were plotted computed between averaged values within microstate masks from FDG-PET SUV, receptor availabilities of $G_{A B A}$, and mGluR5 with the source-localised microstate maps from EEG.

combined trimodal data. For the first time, insight into the neurobiological foundation of the E/I-B (given as the quantification of the availability of the excitatory and inhibitory receptors as well as the glucose metabolism intensity) has been gained alongside the functional connectivity measures and EEG microstates during the RS.

\section{EEG microstates}

The brain network maps of microstates shown in Fig. 2 demonstrate the location of neuronal generators of each microstate, with all microstates showing the highest current density distribution in the posterior and anterior cingulate cortex. Additional neuronal generator distributions were seen in the precuneus, precentral and postcentral gyrus, superior frontal gyrus and paracingulate gyrus, comparable with the previously reported results $^{15,16}$. In addition the spatially overlapping regions are seen in the brain network masks of all microstates as reported previously ${ }^{16}$. A one to one comparison of microstate results presented here against published similar results ${ }^{16}$ cannot be made due to the differences in the EEG data. Custo and colleagues recorded the EEG data in a shielded room with 256 high-density electrodes while in the present study EEG was recorded in MR environment using 32 or 64 channel EEG electrodes. Even though artefact corrections were made, a 100\% accurate removal of residual artefacts due to gradient, cardioballistic and movement cannot be made with the existing state of the art techniques. Also, the voxel size of the

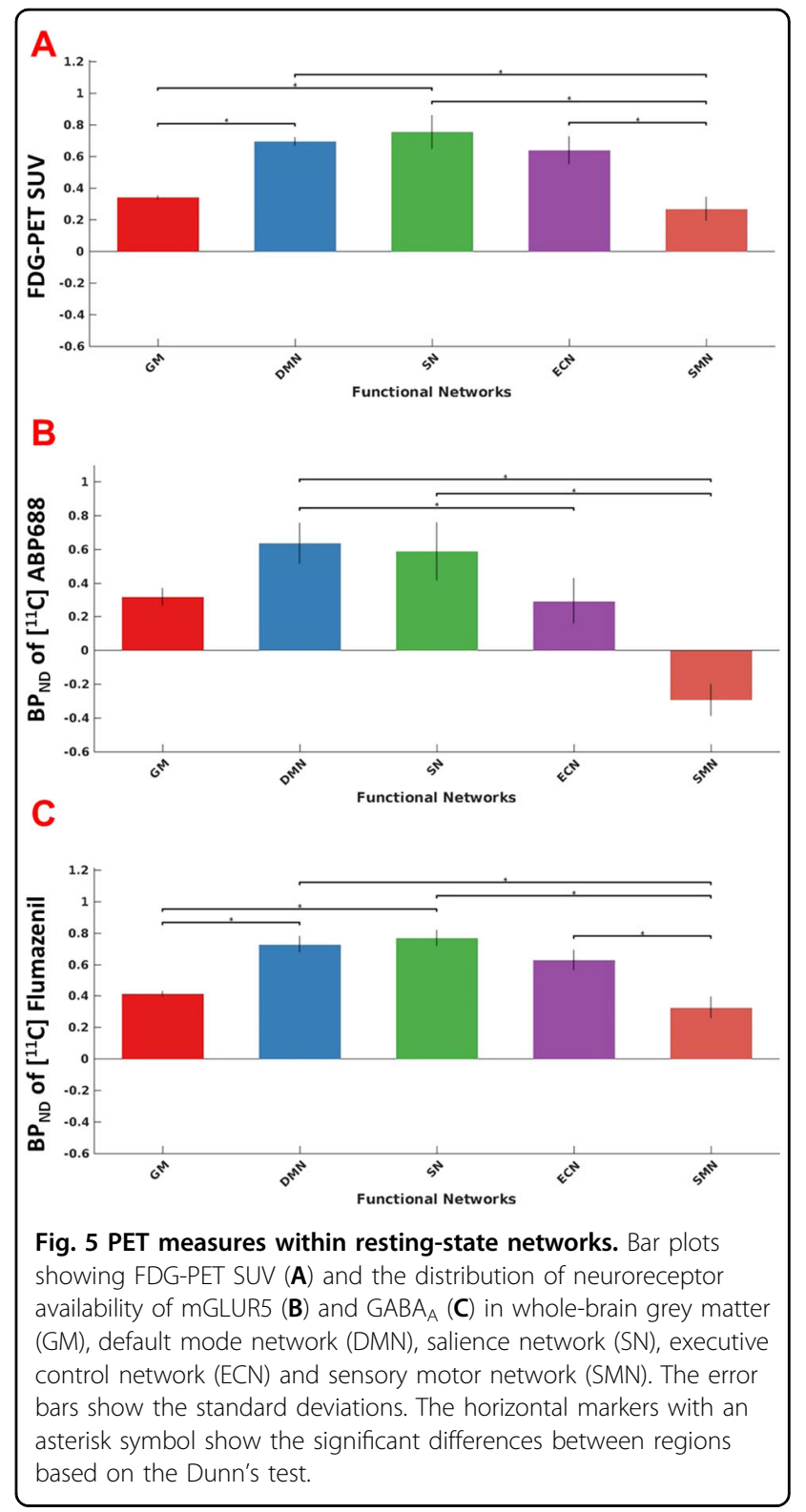

inverse solution space of standard LORETA method used in this study is $5 \mathrm{~mm}^{3}$ which contributed to the spatial overlap of microstate-based brain networks. Notwithstanding to these limitations, the averaged microstatesbased brain network maps represent a fragmented version of the fMRI RSNs (Fig. 2) as already reported ${ }^{15,16}$. In the identified microstates-based brain network masks, the RSN regions were restricted to the cortical areas only usually identified via fMRI or PET. In line with previous observations ${ }^{16,25}$, the source-localised microstates show the major fMRI-RSN regions, observed by means of fMRI or PET imaging, to be a low-pass-filtered version of the microstate dynamics. 


\section{$\mathrm{GABA}_{\mathrm{A}}$ and mGluR5 RA and their associations with EEG microstates}

The results depicted in Fig.1, show the averaged $\mathrm{BP}_{\mathrm{ND}}$ images of FMZ and ABP, corresponding to the distribution of neuroreceptor availability of $\mathrm{GABA}_{\mathrm{A}}$ and mGluR5,

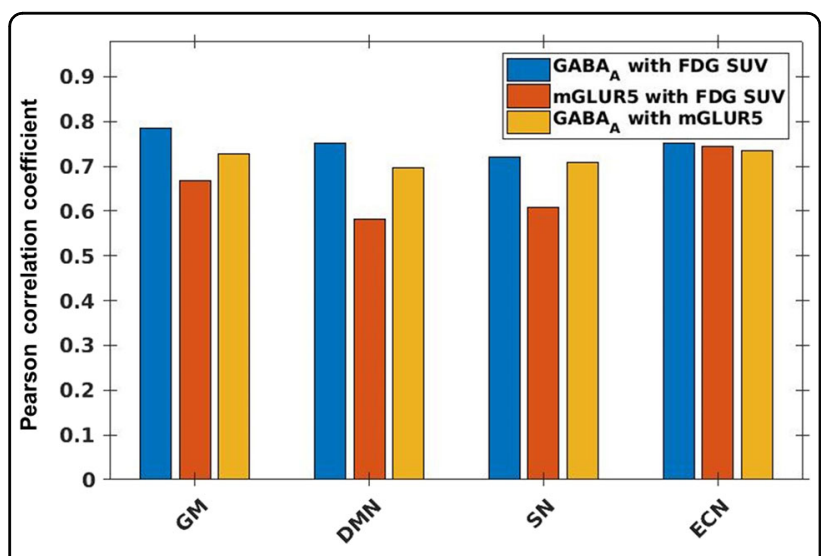

Fig. 6 Correlation values between PET measures. Bar plot showing Pearson correlation coefficient values calculated between receptor availability of GABA with FDG-PET SUV (blue bars), receptor availability of mGluR5 with receptor availability of $\mathrm{GABA}_{A}$ (red bars), and receptor availability of $\mathrm{GABA}_{\mathrm{A}}$ with receptor availability of mGluR5 (yellow bars). The correlation values are shown for the whole-brain grey matter region (GM), the default mode network (DMN), the salience network (SN) and the executive control network (ECN). respectively. These corroborate the notion that $\mathrm{GABA}_{\mathrm{A}}$ receptors are widely distributed throughout the human brain $^{26}$, whereas mGluR5 receptors are mostly distributed in the anterior cingulate cortex, the medial temporal lobe, the putamen and the caudate nucleus ${ }^{27}$.

In addition, the results of this study show a significantly higher RA of $\mathrm{GABA}_{\mathrm{A}}$ in the DMN and $\mathrm{SN}$ compared to whole-brain GM as well as in all of the core RSNs compared to the non-core RSNs (SMN) (Fig. 5C). Thus, even though $\mathrm{GABA}_{\mathrm{A}}$ is widely distributed in the brain, the significantly higher RA in the RSNs suggests greater inhibitory action, particularly during resting conditions.

With respect to the RA of mGluR5, while it was not found to be higher in any of the core RSNs compared to whole-brain GM (Fig. 5B), it was found to be significantly higher in the two core RSNs DMN and SN compared to the SMN (Fig. 5B). Thus, the higher RA of both $\mathrm{GABA}_{\mathrm{A}}$ and mGluR5 receptors in the core RSNs compared to SMN during RS indicates that these networks, in particular, require a stable equilibrium between excitation and inhibitory processes compared to the SMN.

Our results pertaining to the link between the cortical neuronal generators of EEG microstates with the RA of $\mathrm{GABA}_{\mathrm{A}}$ receptors show comparatively higher correlation values than with the FDG-PET SUV and with the RA of mGluR5 receptors (Fig. 4). This is in support of other EEG studies which have shown the involvement of $\mathrm{GABA}_{\mathrm{A}}$

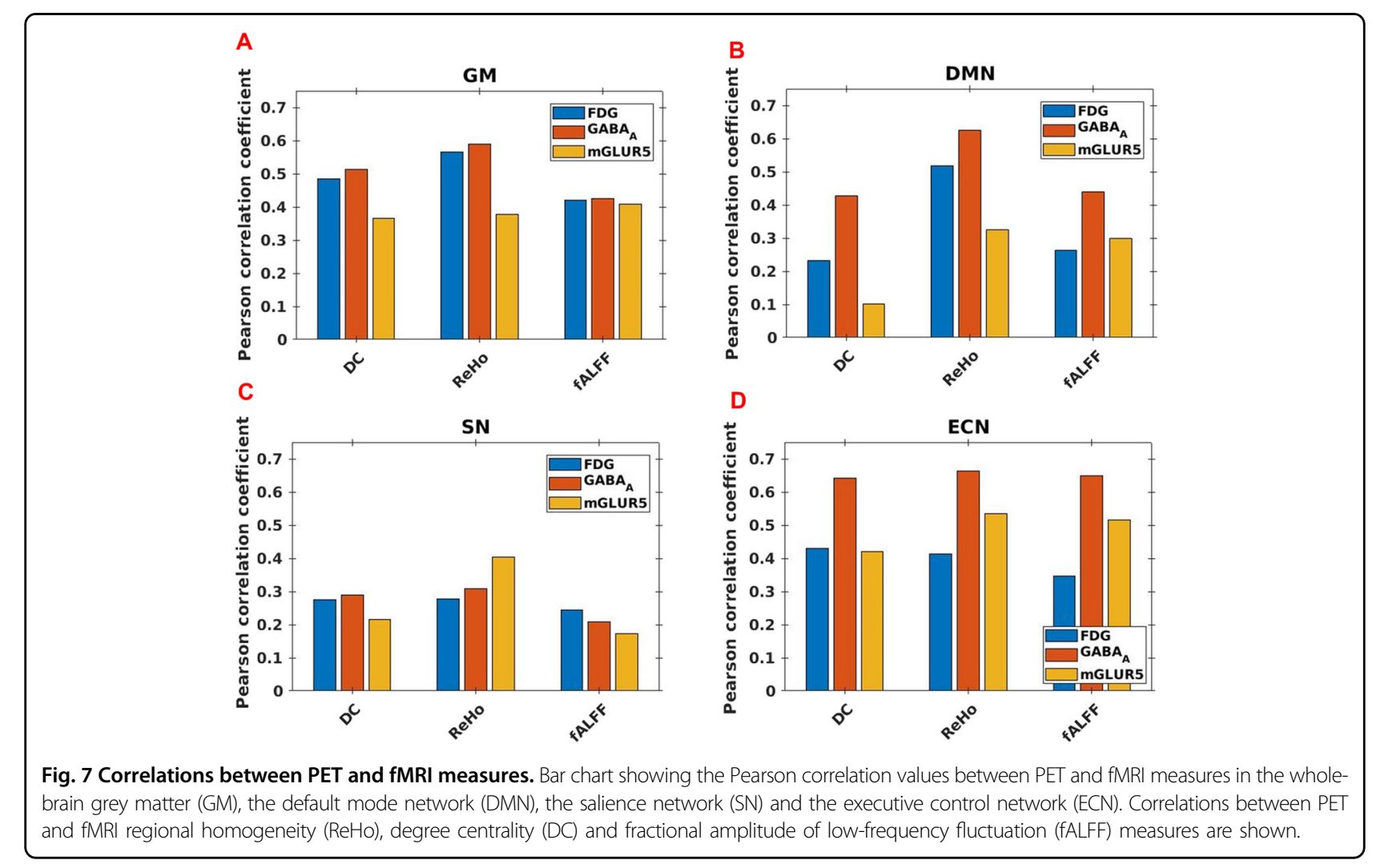


receptors in the beta oscillations $(13-28 \mathrm{~Hz})^{28}$. Furthermore, studies using MRS have also demonstrated an association between GABA concentration and EEG signals in the beta band in the sensorimotor cortex ${ }^{29}$, auditory gating in schizophrenia ${ }^{30}$, and in the working memory load processing capacity in the dorsolateral prefrontal $\operatorname{cortex}^{31}$. As microstates represent a fragmented low-pass-filtered version of the RSNs and considering that $\mathrm{GABA}_{\mathrm{A}}$ receptors mainly mediate synaptic inhibition, our results confirm, from a different perspective, the importance of the inhibitory processes mediated by $\mathrm{GABA}_{\mathrm{A}}$ receptors in the RSNs during rest.

Despite the higher RA of mGluR5 seen in the RSNs (Fig. 5B), the neuronal generators of EEG microstates did not show substantial correlation with the RA of mGluR5 (Fig. 4). mGluR5 receptors are reported to regulate various mechanisms implicated in neurogenesis and synaptic maintenance ${ }^{32}$ as well as in long-lasting forms of synaptic plasticity, including long-term depression (LTD) and long-term potentiation (LTP) ${ }^{33}$. Further, the mGluR5 receptors share a structural and functional link with the NMDA-R and jointly modulate glutamatergic signalling ${ }^{34}$. In studies combining EEG and PET measures, an association between the mGluR5 RA and EEG delta activity could be demonstrated ${ }^{35}$. Keeping in mind the proven association between the glutamate concentration and EEG oscillations in the gamma band ${ }^{36}$, the negligible association between mGluR5 receptors and the neuronal generators of EEG microstates accessed in the lowfrequency range in our study indicate that the excitation of neurons during RS is likely mediated by other glutamate receptors in the $2-20 \mathrm{~Hz}$ frequency range.

\section{Glucose metabolism and its association with the availability of mGlu5 and $\mathrm{GABA}_{\mathrm{A}}$ receptors}

Glucose is the main energy substrate of the adult brain. Although the brain represents only $2 \%$ of the body mass, it requires about $20 \%$ of the oxygen and $25 \%$ of the glucose consumed by the human body ${ }^{37}$. Thereby, the basal metabolism (required for vital functions of neurons) accounts for $30 \%$ of the energy consumed by the brain with the remaining $70 \%$ being accounted for by spontaneous activity ${ }^{38}$.

In our study, we replicate a significantly higher FDG uptake (corresponding to a higher glucose metabolism) in the core RSNs-DMN and the SN-compared to the SMN and the whole-brain GM regions in the RS, as already previously shown in other studies ${ }^{8,38}$. Previous investigations have also demonstrated that, relative to this very high rate of ongoing energy consumption in the RS, the additional energy consumption associated with changes in brain activity is remarkably small, often $<5 \%$ of the baseline level of activity ${ }^{39}$. Our results indicate that the main proportion of the energy consumption during the RS is directed to the highly active DMN and SN. A task-related deactivation of the $\mathrm{DMN}^{40}$ may be permissive for an energy redirection to other task-related networks. The high-energy demand of the SN during rest may be associated with the continuous state of readiness required within the scope of its responsibility for detecting and filtering information necessary to maintain goal-directed behaviour by shifting attention between external and internal processes ${ }^{41}$.

Regarding the question relating to the associations between the glucose metabolism measured via FDG-SUV and the RA of the mGluR5 and GABA $A_{A}$, strong voxellevel correlations were observed with both neuroreceptor types in all examined core RSNs, as well as in the wholebrain GM (Fig. 6).

These results correspond with the established knowledge that glutamate cycling consumes a large proportion of the total energy utilised by the brain ${ }^{42}$, and that $60-80 \%$ of the total energy consumption in the brain during RS is devoted to glutamate cycling ${ }^{43}$. Glutamate is the principal neurotransmitter responsible for the majority of excitatory synaptic activity. However, it exhibits very complex signalling and functional diversity, which is decoded by several different receptor types assigned to the ionotropic (iGluR) and the metabotropic glutamate receptor family (mGluR). In this case, mGluR5 belongs to the group I mGluRs family and is usually localised postsynaptically. In contrast with group II and group III mGluRs, which are often localised on presynaptic terminals or preterminal axons where they inhibit neurotransmitter release, activation of group I mGluRs often leads to cell depolarisation and increases in neuronal excitability ${ }^{44}$. Thus, mGluR5 receptor may be considered as a suitable representative of excitatory glutamatergic neurotransmission and is tightly coupled with glucose metabolism.

We further observed a strong correlation between the $\mathrm{GABA}_{\mathrm{A}}$ receptors, which are widely distributed in the cerebral cortex, and the FDG-PET SUV in the core RSNs and whole-brain GM (Fig. 6). $\mathrm{GABA}_{\mathrm{A}}$ receptors are pentameric ligand-gated ion channels mainly composed of two $\alpha$, two $\beta$ and a single $\gamma$ subunit, which are selected from a diverse pool of 19 different subunit types ${ }^{45}$ and are responsible for most fast synaptic inhibition in the mammalian brain. They also play a crucial role at extrasynaptic sites by mediating tonic inhibition in the brain ${ }^{46}$. The tight association between the glucose homoeostasis and the GABAergic neurotransmission has been demonstrated in some previous PET studies ${ }^{47}$. Here we confirm these findings by demonstrating a tight interrelation between the $\mathrm{GABA}_{\mathrm{A}} \mathrm{RA}$ and glucose metabolism, measured directly, in vivo.

Finally, high correlation values were found between mGluR5 and $\mathrm{GABA}_{\mathrm{A}}$ in all core RSNs as well as in the 
remaining GM (Fig. 6). Considering that these are the two most important representatives of the receptors required for excitatory and inhibitory processes in the brain, our results indicate a neurobiological basis of the E/I-B by the means of the innovative trimodal approach.

\section{Glucose metabolism and its association with EEG microstates}

Several previous studies have demonstrated tight associations between glucose concentration and metabolic rate and EEG signal changes ${ }^{48}$. A direct association between the glucose metabolism and the neuronal generators of EEG-microstates has, to the best of our knowledge, not been investigated before now. In our study, rather surprisingly, no association was observed between the FDG-SUV and the neuronal generators of the four EEG-microstates (Supplementary Table 1). Given that the synaptic currents responsible for neuronal firing (recorded via EEG during RS) display a high-energy demand, mainly provided by glucose, a stronger correlation was expected between the cortical generators of EEG microstates and the glucose consumption indicated by the SUV of FDG. One possible explanation for the rather weak correlation values revealed here may be due to the different observational periods: the FDG-PET SUV values are showing the FDG uptake averaged over about $6 \mathrm{~min}$ while the microstates lasted only a few milliseconds.

\section{Associations of the $\mathrm{fMRI}$ measures with glucose metabolism and mGluR5 and GABA $\mathrm{GA}_{\mathrm{A}}$}

The fMRI techniques utilise the BOLD contrast to map the brain's functional integration. The fMRI signal in the DMN is influenced by the energy demands associated with synaptic currents and action potential propagation ${ }^{42}$. In the absence of external stimuli, the RS-fMRI signal partially represents the metabolic processes but also fluctuations of spontaneous neuronal activity ${ }^{49}$. Thus, examining the relationship between the SUV using FDGPET and fMRI measures will likely facilitate an accurate interpretation of the BOLD signals.

In this study, the RS-fMRI measures (DC, ReHo, and fALFF) showed a significant voxel-level correlation with FDG-PET SUV measures in whole-brain GM (Fig. 7A), which is in agreement with previously reported results ${ }^{50}$. This indicates a high interrelation between metabolic processes and spontaneous neuronal activity-the highenergy demand of the brain seems to be utilised for high functional connectivity (as revealed in DC and ReHo) as well as for the BOLD fluctuations (as revealed by fALFF).

However, the correlation between the fMRI measures and the SUV from FDG-PET is weaker in the core RSNs compared to GM (Fig. 7B-D). This may be explained due to the high-energy efficiency of the RSNs ${ }^{38}$, which, despite having more functional connections, possibly use less energy. Further, our results indicate a stronger association of FDG-PET SUV with ReHo than with DC in the DMN (Fig. 7B). This may be on account of the more shortrange, energy-consuming connections ( $\mathrm{ReHo}$ ) within the DMN or a higher energy-efficiency of the longer-range connections (DC). Such difference was not observed within the SN and the ECN, where within both networks the correlation of the FDG-PET SUV values with ReHo and DC was similarly strong. This points towards a unique property of the DMN relating to energetic efficiency.

To date, the association between the fMRI measures and the glutamatergic and GABAergic neurotransmission has primarily been investigated using the single-voxel magnetic resonance spectroscopy (MRS) technique. In task conditions, depending on the brain regions and cognitive processes involved, GABA levels have shown both positive and negative correlations with the BOLD signal $^{51,52}$ Arrubla et al. ${ }^{53}$ have found GABA concentration in the posterior cingulate cortex (PCC) to be inversely correlated with connectivity within the DMN during $\mathrm{RS}^{53}$. Furthermore, both resting-state and task-based fMRI studies $^{2,54}$ have also shown associations with glutamate concentration levels. However, the drawback of single-voxel MRS is that the neurotransmitter's concentration from a single voxel (typically a $3 \mathrm{~cm}$ isotropic cube) is used to investigate connectivity within RSNs or the whole brain. In simultaneous MR-PET imaging, this drawback is surmounted by calculating three-dimensional parametric images of the whole brain (about $3 \mathrm{~mm}$ resolution), which enables region-of-interest (ROI) analysis.

Our results show disparate association patterns between the fMRI measurements and the $\mathrm{GABA}_{\mathrm{A}}$ and mGluR5 RA for the three core RSNs and GM, as depicted in Fig. 7.

Regarding the associations between the long-range connectivity measure (DC) and the excitatory and inhibitory RA (mGluR5 and $\mathrm{GABA}_{\mathrm{A}}$ ), the most prominent difference was observed in the DMN. Here we found an almost fourfold higher association with the $\mathrm{GABA}_{\mathrm{A}} \mathrm{RA}$ than with the mGluR5 RA. A similarly directed, albeit lower relation, was also observed for the parameters ReHo and $\mathrm{fALFF}$, indicating a predominant role of the inhibitory GABAergic neurotransmission within the E/I-B in the DMN.

Interestingly, a higher association between the shortrange measure ReHo and the mGluR5 RA was only observed in the SN. The $\mathrm{SN}$ is considered to be a filtration and amplification network that is responsible for evaluating specific external/internal stimuli and assigning the relevance of stimuli for goal-directed behaviour $^{55}$. In this specific operating range, the $\mathrm{SN}$ may request a higher capacity of excitatory (compared to the inhibitory) receptors and connections than within other RS networks. 


\section{Summary}

In summary, the results presented above contribute to a better understanding of the questions initially raised in the "Introduction" section and reveal the following pertinent conclusions:

1. The main, relatively high, significant correlations of all four EEG microstates were observed with the RA of $\mathrm{GABA}_{\mathrm{A}}$ receptors, followed by considerably lower correlations with the FDG-PET SUV and correlations with the RA of mGluR5 receptors to a very low extent. Considering that microstates represent a fragmented low-pass-filtered version of the RSNs, these results confirm the importance of the inhibitory processes mediated by $\mathrm{GABA}_{\mathrm{A}}$ receptors in the electrophysiological activity of the RSNs during RSs. The negligible association between mGluR5 receptors and the neuronal generators of EEG microstates accessed in the low-frequency range indicate that the excitatory contribution in the E/I-B may be mediated by other glutamate receptors than mGluR5.

2. A significantly higher distribution of $\mathrm{GABA}_{\mathrm{A}}$ and mGluR5 receptors was observed in both the DMN as well as in the SN compared to the non-core RSNs (SMN). However, compared to the whole GM, only the RA of $\mathrm{GABA}_{\mathrm{A}}$ was significantly higher in these networks. This finding once again supports a leading role of the inhibitory processes during the RS. Furthermore, the RA of mGluR5 and GABA $_{\mathrm{A}}$ correlated significantly with each other in all core RSNs and in the remaining GM, indicating the general importance of E/I-B during the RS.

3. The RA of the mGluR5 and $\mathrm{GABA}_{\mathrm{A}}$ receptors both showed strong voxel-level correlations with the glucose metabolism measured via FDG-SUV in all examined core RSNs as well as in the whole-brain GM. This finding indicates that the high-energy demand during the RS may be mainly directed to the maintenance of the E/I-B.

4. Each of the observed core RSNs and GM showed specific association patterns between the different fMRI measure's and the $\mathrm{GABA}_{\mathrm{A}}$ and mGluR5 RA. Thus, the relative proportion of excitatory and inhibitory processes within the E/I-B seems to differ between the single RSNs and is most likely to be dependent on their main functions.

\section{Limitations and outlook}

Several methodological limitations should be kept in mind when interpreting our results. One of the main limitations of the study is the relatively small sample size, a fact hampering a broader generalisation of our findings. Due to the complex and high-cost procedures involved in the simultaneous trimodal measurements and invasive nature of PET imaging, recruiting a larger sample size is a major challenge. Furthermore, the investigation combines data from three separate studies, each using just one PET tracer in a separate subject group. However, conducting all three examinations in the same group is not conceivable due to the complexity of the trimodal data acquisition paradigms as well as due to the ethical and legal implications relating to the repeated radiation exposure from PET imaging.

RA was only examined in mGluR5 and $\mathrm{GABA}_{\mathrm{A}}$ receptors, which represent a small fraction of the excitatory and inhibitory neuroreceptors. Investigating all neuroreceptors, or at least the most prominent ones, would have helped to delineate the mechanisms behind the neurovascular and neuroelectric signals in greater detail. Nevertheless, the two chosen receptor types appear to be the most representative of the receptors required for excitatory and inhibitory neurotransmission.

Further, the radioligands used for the quantification of the mGluR5 and $\mathrm{GABA}_{\mathrm{A}} \mathrm{RA}$ differ in their receptor binding properties (for ABP: dissociation constant $\left(K_{\mathrm{D}}\right)=$ $1.7 \pm 0.2 \mathrm{nmol} / \mathrm{L}^{56}$; for FMZ $K_{\mathrm{D}}=0.7 \pm 0.26 \mathrm{nM}^{57}$ ).

These limitations notwithstanding, the results presented here give new insight into the neurobiological foundation of the E/I-B (given as the quantification of the availability of the excitatory and inhibitory receptors as well as the glucose metabolism intensity) in terms of functional connectivity measures and EEG microstates during the RS. Further research involving healthy individuals as well as patients with mental illness will likely shed valuable light towards a better understanding of the neurobiological alterations underlying specific diseases.

\section{Methods}

The trimodal data presented in this study were acquired using three different PET-radiotracers: ABP, FMZ and FDG. For readability, the datasets are hereafter referred to as ABP, FMZ and FDG data, based on the radiotracer used in the study.

\section{Trimodal data acquisition}

The simultaneous trimodal data were acquired using a $3 \mathrm{~T}$ hybrid MR-BrainPET scanner system (Siemens, Erlangen, Germany) ${ }^{58}$ equipped with an MR-compatible EEG system (Brain Products, Gilching, Germany). As shown in a previous study, the EEG electrodes used in this system do not cause any noticeable effect on PET images $^{59}$. The study was approved by the Ethics Committee of the Medical Faculty of RWTH Aachen University and the German Federal Office for Radiation Protection (Bundesamt für Strahlenschutz). All methods were performed according to the relevant guidelines and regulations. The study was conducted in accordance with the Declaration of Helsinki and prior written consent was obtained from 
Table 1 Subject and injected activity details for each study.

\begin{tabular}{llll}
\hline Study & Number of subjects & Mean age in years in years & Injected activity in MBq \\
\hline ABP & 9 (males) & $23 \pm 3$ & $407 \pm 56\left(\mathrm{~K}_{\mathrm{BO}}=61.79 \mathrm{~min}\right)$ \\
FMZ & 10 (males) & $26 \pm 3.72$ & $411 \pm 18\left(\mathrm{~K}_{\mathrm{Bol}}=46.22 \mathrm{~min}\right)$ \\
FDG & 10 (males) & $28 \pm 4$ & $200 \pm 30$ \\
\hline
\end{tabular}

all volunteers. Moreover, the German Federal Office for Radiation Protection has imposed a 10-year restriction on the injection of PET tracers for the second time in healthy individuals for research purposes. To reduce the implications of this limitation, we included very well-matched groups in the three separate investigations.

Only the trimodal FDG data presented in this study were taken from a previously published work ${ }^{8}$. Only subjects (or their first degree relatives) with no history of neuropsychiatric disorders as assessed via Mini International Neuropsychiatry Interview $(\mathrm{MINI})^{60}$ were included in each study. In addition, only male subjects were included due to the effects of the menstrual cycle on variations in neurotransmitter levels ${ }^{61}$. The data and results presented in this manuscript will be available upon request via the corresponding author.

The data acquisition protocol for each modality is described below.

\section{PET data acquisition}

Radiosynthesis of APB and FMZ were performed according to literature ${ }^{62,63}$. The PET radioligand was injected as bolus in the FDG study and as bolus plus infusion in the ABP and FMZ studies. The radiotracer was injected via an intravenous (IV) line while the subject was lying in the $3 \mathrm{~T}$ hybrid MR-BrainPET scanner. Details relating to the number of subjects, age, and injected activity with $K_{\mathrm{Bol}}$, for each study are given in Table 1 . PET data acquisition in list mode started immediately following injection of the tracer. In ABP and FMZ studies, the eyes closed RS-fMRI and EEG data were simultaneously recorded from the time the radiotracer reached equilibrium in the brain. In the FDG study where no equilibrium is reached, the RS data were recorded exactly from $50 \mathrm{~min}$ after radiotracer injection in all subjects. During RS data acquisition, subjects were instructed to close their eyes and not to fall asleep. The simultaneous acquisition of RS data lasted for $\sim 6 \mathrm{~min}$.

The PET data were iteratively reconstructed (3D OPOSEM, 32 iterations and 2 subsets) into 2-min frames. Attenuation correction was performed based on an MRbased and template-based procedure ${ }^{64}$. In addition, the PET data reconstruction incorporated corrections for random and scattered coincidences, dead time, radioactive decay, and pile up. The reconstructed image frames comprised $256 \times 256$ pixels and 153 slices (voxel size $1.25 \mathrm{~mm}^{3}$, isotropic).

\section{MR data acquisition}

In all investigations, the anatomical and RS-fMRI images were acquired using magnetisation-prepared rapid acquisition gradient-echo (MP-RAGE) and T2*-weighted echo planar imaging (EPI) sequences, respectively. The anatomical images were acquired before the RS data acquisition during the same session. The MR-imaging parameters considered in each sequence are given below:

- MP-RAGE sequence: repetition time (TR) $=2250 \mathrm{~ms}$, echo time (TE) $=3.03 \mathrm{~ms}$, field-of-view (FOV) $256 \times$ $256 \times 176 \mathrm{~mm}^{3}$, voxel size $=1 \times 1 \times 1 \mathrm{~mm}^{3}$, flip angle $(\mathrm{FA})=9^{\circ}, 176$ sagittal slices and a GRAPPA acceleration factor of 2 with 70 auto-calibration signal lines.

- EPI sequence (for FDG and FMZ studies): $\mathrm{TR}=$ $2200 \mathrm{~ms}, \mathrm{TE}=30 \mathrm{~ms}, \mathrm{FOV}=200 \times 200 \times 108 \mathrm{~mm}^{3}$, voxel size $=3.125 \times 3.125 \times 3.0 \mathrm{~mm}^{3}, \quad F A=80^{\circ}$, number of slices $=36$, number of volumes $=169$.

- EPI sequence (for ABP study): $\mathrm{TR}=2000 \mathrm{~ms}, \mathrm{TE}=$ $30 \mathrm{~ms}, \mathrm{FOV}=192 \times 192 \times 108 \mathrm{~mm}^{3}$, voxel size $=3 \times$ $3 \times 3.0 \mathrm{~mm}^{3}, \quad F A=80^{\circ}, \quad$ number of slices $=33$, number of volumes $=180$.

\section{EEG data acquisition}

Prior to the trimodal acquisition, the MR-compatible EEG cap (BrainCap MR, EasyCap GmbH, Herrsching, Germany) was placed on the subject's head and an electrolyte gel (ABRALYT 2000, EASYCAP GmbH, Herrsching, Germany) was applied between the EEG electrodes and the scalp to reduce impedance. The impedance of all recording electrodes was kept below $10 \mathrm{k} \Omega$. A 32-channel EEG cap was used in the FDG study and a 64-channel EEG cap was used in the ABP and FMZ studies. The EEG electrodes were positioned according to the 10-20 system in both caps. The electrocardiogram (ECG) signals were recorded using an additional electrode. The ECG electrode was connected to the distal part of the trapezius muscle. The RS EEG data (eyes closed) and ECG were recorded using the Brain Vision Recorder (Brain Products, Gilching, Germany) while the subject lay in the scanner. The subjects were instructed to stay calm and relaxed and not fall asleep during the RS 
measurements. The RS EEG data were recorded at a sampling rate of $5 \mathrm{kHz}$ and bandwidth between 0.016 and $250 \mathrm{~Hz}$. The helium pump of the MR system was switched off during trimodal RS data acquisition in order to avoid the effects of vibration on the MR and EEG system as well as on the subjects.

\section{Data analysis \\ PET}

The PET images were analysed using the PMOD (version 3.5) software package. The reconstructed PET images were corrected for motion using a PET image-based motion correction method ${ }^{65}$. Following motion correction, the PET images were smoothed with a Gaussian kernel size of $2.5 \mathrm{~mm}$ along all three directions. Only the three PET image frames corresponding to the RS measurement were considered for further analysis.

The parametric images representing the $\mathrm{BP}_{\mathrm{ND}}{ }^{7}$ in each subject were calculated from the reconstructed $\mathrm{ABP}$ and FMZ PET data. The three PET image frames were averaged (time weighted). The $\mathrm{BP}_{\mathrm{ND}}$ was calculated as $\left(C_{\mathrm{T}}-C_{\mathrm{REF}}\right) / C_{\mathrm{REF}}$, where $C_{\mathrm{T}}$ denotes the activity concentration in an area of high neuroreceptor density, and $C_{\mathrm{REF}}$ denotes activity concentration in a non-specificbinding region (region with very low receptor density). The cerebellum ${ }^{66}$ and pons ${ }^{67}$ were chosen as $C_{\mathrm{REF}}$ regions for $\mathrm{ABP}$ and FMZ, respectively. The $C_{\mathrm{REF}}$ regions were defined using a three-dimensional maximum probability atlas $^{68}$ on the MNI space co-registered anatomical images (MP-RAGE).

The parametric images representing the SUV were calculated from FDG-PET data using the averaged PET image frame during RS. The total activity injected (MBq) and the body weight $(\mathrm{kg})$ of the subject were accounted for when calculating the SUV. The final unit of SUV is $\mathrm{g} / \mathrm{ml}$.

\section{fMRI}

The RS-fMRI measures (ReHo, DC, fALFF) were calculated using MATLAB-based software packages, SPM12 (http://www.fil.ion.ucl.ac.uk/spm/) and DPABI ${ }^{69}$. The pre-processing steps included the removal of the first 10 volumes of the total acquisition ${ }^{70}$, slice-timing correction with respect to the middle slice of the functional image, realignment, and nuisance covariates regression (NCR). The covariates for NCR included head motion parameters $^{71}$, whole-brain white matter (WM) and cerebrospinal fluid (CSF) mean signals, and the constant, linear and quadratic trends in the BOLD signals. Following the pre-processing steps, RS-fMRI measures were calculated in the subject's native image space. The temporal filtering of BOLD fMRI signals between 0.01 and $0.08 \mathrm{~Hz}$ was performed before DC and ReHo calculation. The ReHo measure was calculated using Kendall's coefficient of concordance $(\mathrm{KCC})^{72}$ as the homogeneity measure over 27 neighbouring voxels ${ }^{73}$. The DC measure was calculated with the Pearson correlation threshold of $0.25(p=0.001)$. The fALFF measure was calculated within the BOLD low-frequency range between 0.01 and $0.1 \mathrm{~Hz}$.

Of the currently identifiable RSNs, the DMN, the salience network (SN) and the executive control network $(\mathrm{ECN})^{74}$ are considered to be the core neurocognitive RSNs and, as such, form the basis of analysis in this study. Furthermore, for the purposes of RA comparison within the RSNs, the sensory motor network (SMN) (a non-core RSN) is also considered as a control region.

\section{EEG}

The EEG data were processed using the MATLABbased open-source software packages EEGLAB (version 13, https://sccn.ucsd.edu/eeglab/index.php) ${ }^{75}$, and FieldTrip software package (http://www.fieldtriptoolbox.org//) and analysed in three steps. First, the data pre-processing was performed to remove artefacts, then the microstates were calculated, and in the final step the microstates were source-localised. Following is a detailed explanation of the EEG-processing steps.

EEG data pre-processing The recorded EEG data were imported to EEGLAB and downsampled to $2048 \mathrm{~Hz}$. When recorded alongside fMRI, EEG data are heavily contaminated by gradient artefacts (GA) due to the switching of the gradient magnets in the MR system. Here, the GA were corrected using the FASTR tool ${ }^{77}$ implemented in EEGLAB. The data were then downsampled to $1000 \mathrm{~Hz}$, filtered between 2 and $20 \mathrm{~Hz}$ using a Hamming windowed sinc finite-impulse response (FIR) filter implemented in the EEGLAB function eegfiltnew, and re-referenced to the average reference. Bad channels were identified and removed based on their correlation to neighbouring channels using the EEGLAB function clean_rawdata. In order to remove non-stationary artefacts, such as head movement, artefact subspace reconstruction $(\mathrm{ASR})^{78}$ was performed with the recommended parameters ${ }^{79}$. Following ASR, the data were again re-referenced to the average reference. The pulsatile flow of blood due to the pumping action of the heart causes ballistocardiogram (BCG) artefacts in the EEG data. An adaptive optimal basis set algorithm $(\mathrm{aOBS})^{80}$, which accounts for the key shortcomings of the commonly used optimal basis set (OBS) algorithm ${ }^{77}$, was used to detect and remove these artefacts from the EEG signal. An automatic blind source separation algorithm was used to remove ocular artefacts ${ }^{81}$. To remove further residual artefacts, independent component analysis (ICA) ${ }^{82}$ was performed and the multiple artefact rejection algorithm (MARA) ${ }^{83}$ was used to classify and remove artefactual components. Further BCG-related components were 
identified and removed with the projection onto independent components (PROJIC) algorithm ${ }^{84}$. Additionally, EEG data showing residual artefacts due to motion were marked and removed using pop_rejcont() EEGLAB function. Finally, the first $30 \mathrm{~s}$ of the RS-EEG data were rejected, as was the case in the fMRI analysis. In order to reduce the computation time, the EEG data were again downsampled to $125 \mathrm{~Hz}$.

Microstate calculation The microstates in the artefactcorrected EEG data were analysed using the Microstate plugin (http://www.thomaskoenig.ch/index.php/software/ microstates-in-eeglab) implementation in EEGLAB. Here, the global brain activity is represented in the global field power (GFP) of the multichannel EEG data ${ }^{85}$, with the EEG topographies being considered to be stable around the maxima of the GFP (GFP peaks) ${ }^{11}$. Thus, the GFP was first calculated for each subject, and the EEG topographic maps were then plotted for GFP peaks. All EEG topographic maps marked as GFP peaks were extracted and submitted for spatial clustering, which was performed using a modified atomised and agglomerate hierarchical clustering (AAHC) algorithm ${ }^{86}$. The AAHC algorithm was set to identify the four dominant EEG topographic maps for each subject. Finally, the EEG topographic maps identified for each subject were also sorted into microstates $\mathrm{A}-\mathrm{D}$, using the published template maps ${ }^{11}$.

Following the microstate calculation and sorting, the EEG data corresponding to each microstate were epoched separately as EEG-MS A, EEG-MS B, EEG-MS C, and EEG-MS D for every individual subject in each study. The epoched microstates of each class was concatenated to give continuous EEG data of particular microstate.

Source localisation The EEG data of each microstate were exported to the LORETA-KEY software (http:// www.uzh.ch/keyinst/loreta.htm) to solve the inverse problem (source localisation). In a first step, the EEG electrode positions in the MNI152 template were determined $^{87}$ and a 3D cortical current density distribution from the EEG data epochs was calculated using sLOR$\mathrm{ETA}^{13}$. In order to solve the inverse problem, sLORETA computations were made in the realistic head model ${ }^{88}$ using the Montreal Neurological Institute template $\left(\right.$ MNI152) ${ }^{89}$. The 3D solution space in sLORETA was restricted to the cortical GM. The inverse solutions showing the cortical current density was averaged for each microstate, resulting in a 3D cortical current density distribution for each microstate from every subject.

\section{Intermodality comparison}

In order to perform intermodality comparisons, the calculated measures from PET, fMRI and EEG MS A-D were co-registered to the MNI152 $\left(2 \times 2 \times 2 \mathrm{~mm}^{3}\right)$ standard space. Additionally, the co-registered images were linearly standardised into $Z$-values ${ }^{50}$ for each subject. $Z$ value standardisation was calculated by subtracting the mean whole-brain voxel value from each voxel and then dividing the difference by the standard deviation of the whole brain. The $Z$-value standardised measures were then smoothed with a Gaussian kernel size of $3 \mathrm{~mm}$ along all three directions.

\section{RSNs definition}

Masks of the RSNs were obtained from an atlas of 90 functional regions of interest (fROIs) ${ }^{90}$. In order to further restrict the analysis to the GM regions, only the voxels within the GM region of the RSNs (which show more than $50 \%$ probability of being GM) were considered (GM correction). A GM tissue- segmented MNI152 $(2 \times 2 \times$ $2 \mathrm{~mm}^{3}$ ) template (GM mask) was used for GM correction. A whole-brain GM mask was created by considering only the voxels that showed more than $50 \%$ probability of being GM from the tissue-segmented MNI152 template (Supplementary. Fig. 1). Given that different subjects were used in each study (FDG, FMZ and ABP), a subject-level comparison of PET values within each calculated PET measure is not possible. Thus, all the SUV and BPND images were averaged, yielding an averaged PET measure image. Similarly, for fMRI measures, and in order to improve the signal-to-noise ratio, averaged fMRI measure images were calculated for DC, ReHo and fALFF for each study. Subsequently, all voxel values within the GMcorrected RSNs and GM masks were extracted from the previously calculated subject-level PET, averaged PET and averaged fMRI measures.

Since the 3D solution space in SLORETA was restricted to only the cortical GM, a separate mask for each microstate (MS mask) was created considering only the voxels in the 3D solution space. The brain networks of each microstate were identified using topographic electrophysiological state source-imaging (TESS) method ${ }^{16,91}$. For this purpose the artefact-corrected continuous EEG data was source localised as mentioned above and the time course of each microstate (microstate dynamics) was estimated. The microstate time course along with the GFP was considered as regressors and fitted to the each of the voxel time series of EEG inverse solution using general linear modelling (GLM) to identify the location of neural sources active during each microstate ${ }^{16}$. The preprocessing steps and TESS method to identify microstate networks are shown in Supplementary Fig. 2. The $p$-values of the estimated beta values for each microstate regressors were corrected for false discovery rate at the significance level of 0.01 using Benjamini and Hochberg procedure ${ }^{92}$. Only the significant beta values of each microstate in every subject was transformed to $Z$-score and 
co-registered to the MNI152 $\left(2 \times 2 \times 2 \mathrm{~mm}^{3}\right)$ standard space. The mask of individual microstate network was identified by considering only the $Z$-score transformed voxels which are more than zero in at least $50 \%$ of the subjects. This resulted in a group mask of each microstate network. The 3D cortical current density distribution of each microstate across subjects were averaged individually for each study. Finally, all voxel values within the MS masks were extracted from the previously averaged PET measures and 3D cortical current density distribution of each EEG microstates.

\section{Statistics}

All statistical analyses were performed using the MATLAB (Release 2017, The MathWorks, Inc., Natick, United States) software package. The mean values of SUV and $\mathrm{BP}_{\mathrm{ND}}$ were calculated from each subject's PET image (FDG, FMZ and ABP) for all of the considered regions (GM, DMN, SN, ECN, SMN and MS masks). In order to compare the differences in each PET measure within the considered regions, a stepdown Dunn's test for non-parametric multiple comparisons $^{93}$ was performed with a significance level of $5 \%$. Dunn's test was performed using a MATLAB implementation $^{94}$. A one-sample Kolmogorov-Smirnov test was conducted to verify the normality of the extracted voxel values from the averaged PET, fMRI and EEG measures. To determine the association between the SUV of the FDGPET and the RA of mGluR5 and $\mathrm{GABA}_{\mathrm{A}}$, Pearson linear correlation coefficients $(r)$ were computed with a significance level of $1 \%$ using all voxel values extracted from the averaged PET measures in GM and the core RSNs. Further, in order to elucidate the relationship between the PET measures and RS-fMRI and source-localised microstates, Pearson linear correlation coefficients $(r)$ were computed with a significance level of $1 \%$ using averaged measures from each study. In all of the Pearson correlation analyses, the family-wise error rate (FWER), due to multiple comparisons, was controlled for using a permutation test ${ }^{95}$. $10^{5}$ Permutations were performed for each comparison (correlation), and the $p$-value was adjusted using the "max statistics" method ${ }^{95}$.

\section{Acknowledgements \\ We thank Dr. Jorge Arrubla for his assistance in FDG trimodal human data acquisition. We are grateful to Dr. Andreas Matusch for his guidance with metabolite correction for PET imaging. We are also thankful to Margo Kersey and Claire Rick for proofreading the manuscript and to Andrea Muren, Cornelia Frey, Silke Frensch and Suzanne Schaden for their technical assistance. The study was partly supported by the EU FP7-funded project TRIMAGE (No. 602621).}

\section{Author details}

'Institute of Neuroscience and Medicine 4, INM-4, Forschungszentrum Jülich, Jülich, Germany. ${ }^{2}$ Department of Psychiatry, Psychotherapy and Psychosomatics, RWTH Aachen University, Aachen, Germany. ${ }^{3}$ JARA-BRAIN, 52074 Aachen, Germany. ${ }^{4}$ Department of Electrical and Computer Engineering, McMaster University, Hamilton, ON, Canada. ${ }^{5}$ Department for Psychiatry, Psychotherapy and Psychosomatics Social Psychiatry, University Hospital of
Psychiatry Zurich, Zurich, Switzerland. ${ }^{6}$ Institute of Neuroscience and Medicine 5, INM-5, Forschungszentrum Jülich, Jülich, Germany. ${ }^{7}$ Department of Nuclear Medicine, RWTH Aachen University, Aachen, Germany. ${ }^{8}$ Division of Clinical Cognitive Sciences, RWTH Aachen University, Aachen, Germany. Institute of Neuroscience and Medicine 11, INM-11, Forschungszentrum Jülich, Jülich, Germany

Funding

Open Access funding enabled and organized by Projekt DEAL.

Conflict of interest

The authors declare that they have no conflict of interest.

Publisher's note

Springer Nature remains neutral with regard to jurisdictional claims in published maps and institutional affiliations.

Supplementary information accompanies this paper at (https://doi.org/ 10.1038/s41398-020-01160-2).

Received: 6 October 2020 Revised: 2 December 2020 Accepted: 8 December 2020

Published online: 18 January 2021

\section{References}

1. Zeki, S. \& Shipp, S. The functional logic of cortical connections. Nature https:// doi.org/10.1038/335311a0 (1988).

2. Duncan, N. W., Wiebking, C. \& Northoff, G. Associations of regional GABA and glutamate with intrinsic and extrinsic neural activity in humans-a review of multimodal imaging studies. Neurosci. Biobehav. Rev. 47, 36-52 (2014).

3. Carcea, I. \& Froemke, R. C. Cortical plasticity, excitatory-inhibitory balance, and sensory perception. Prog Brain Res 207, 65-90. https://doi.org/10.1016/B978-0444-63327-9.00003-5 (2013).

4. Allen, P., Sommer, I. E., Jardri, R., Eysenck, M. W. \& Hugdahl, K. Extrinsic and default mode networks in psychiatric conditions: relationship to excitatoryinhibitory transmitter balance and early trauma. Neurosci. Biobehav. Rev. https://doi.org/10.1016/j.neubiorev.2019.02.004 (2019).

5. Logothetis, N. K. What we can do and what we cannot do with fMRI. Nature 453, 869-878 (2008).

6. Sawle, G. V. \& Brooks, D. J. Positron emission tomography studies of neurotransmitter systems. J. Neurol. 237, 451-456 (1990).

7. Mintun, M. A., Raichle, M. E., Kilbourn, M. R., Wooten, G. F. \& Welch, M. J. A quantitative model for the in vivo assessment of drug binding sites with positron emission tomography. Ann. Neurol. https://doi.org/10.1002/ ana.410150302 (1984).

8. Shah, N. J. et al. Multimodal fingerprints of resting state networks as assessed by simultaneous trimodal MR-PET-EEG imaging. Sci. Rep. 7, 6452 (2017).

9. Rajkumar, R. et al. Comparison of EEG microstates with resting state fMRI and FDG-PET measures in the default mode network via simultaneously recorded trimodal (PET/MR/EEG) data. Hum. Brain Mapp. https:/doi.org/10.1002/ hbm.24429 (2018).

10. Lehmann, D. Past, present and future of topographic mapping. Brain Topogr. 3, 191-202 (1990).

11. Koenig, T. et al. Millisecond by millisecond, year by year: normative EEG microstates and developmental stages. Neuroimage 16, 41-48 (2002).

12. Khanna, A., Pascual-Leone, A., Michel, C. M. \& Farzan, F. Microstates in restingstate EEG: current status and future directions. Neurosci. Biobehav. Rev. 49, 105-113 (2015).

13. Pascual-Marqui, R. D. Standardized low-resolution brain electromagnetic tomography (sLORETA): technical details. Methods Find. Exp. Clin. Pharmacol. 24(Suppl D), 5-12 (2002).

14. Van De Ville, D., Britz, J. \& Michel, C. M. Nikos logothetis by K. EEG microstate sequences in healthy humans at rest reveal scale-free dynamics. PNAS 107, 18179-18184 (2010).

15. Britz, J., Van De Ville, D. \& Michel, C. M. BOLD correlates of EEG topography reveal rapid resting-state network dynamics. Neuroimage 52, 1162-1170 (2010).

16. Custo, A. et al. Electroencephalographic resting-state networks: source localization of microstates. Brain Connect. 7, 671-682 (2017). 
17. Treyer, V. et al. Evaluation of the metabotropic glutamate receptor subtype 5 using PET and 11C-ABP688: assessment of methods. J. Nucl. Med. 48, 1207-1215 (2007).

18. Hansen, T. D., Warner, D. S., Todd, M. M., Baker, M. T. \& Jensen N. F. The influence of inhalational anesthetics on in vivo and in vitro benzodiazepine receptor binding in the rat cerebral cortex. Anesthesiology https:/doi.org/ 10.1097/00000542-199101000-00016 (1991).

19. Lecrux, C. et al. Pyramidal neurons are 'neurogenic hubs' in the neurovascular coupling response to whisker stimulation. J. Neurosci. https://doi.org/10.1523/ JNEUROSCI.4943-10.2011 (2011)

20. Just, N. \& Sonnay, S. Investigating the role of glutamate and GABA in the modulation of transthalamic activity: a combined fMRI-fMRS Study. Front. Physiol. https://doi.org/10.3389/fphys.2017.00030 (2017).

21. Lee, M. H., Smyser, C. D. \& Shimony, J. S. Resting-state fMRl: a review of methods and clinical applications. Am. J. Neuroradiol. 34, 1866-1872 (2013).

22. Zang, Y., Jiang, T., Lu, Y., He, Y. \& Tian, L. Regional homogeneity approach to fMRI data analysis. Neuroimage 22, 394-400 (2004).

23. Buckner, R. L. et al. Cortical hubs revealed by intrinsic functional connectivity: mapping, assessment of stability, and relation to Alzheimer's disease. J. Neurosci. 29, 1860-1873 (2009).

24. Zou, Q. H. et al. An improved approach to detection of amplitude of lowfrequency fluctuation (ALFF) for resting-state fMRI: fractional ALFF. J. Neurosci. Methods 172, 137-141 (2008).

25. Pascual-Marqui, R. D. et al. The resting microstate networks (RMN): cortical distributions, dynamics, and frequency specific information flow. Preprint at arxiv: 1411.1949, https://doi.org/10.5167/uzh-100596 (2014).

26. Waldvogel, H. J., Baer, K. \& Faull, R. L. M. Distribution of GABAA receptor subunits in the human brain. In GABA and Sleep: Molecular, Functional and Clinical Aspects (eds Monti, J. M., Pandi-Perumal, S. R. \& Möhler, H.) 73-93 (Springer Basel, Basel, 2010).

27. Ametamey, S. M. et al. Human PET studies of metabotropic glutamate receptor subtype 5 with ${ }^{11}$ C-ABP688. J. Nucl. Med. 48, 247-252 (2007).

28. Porjesz, B. et al. Linkage disequilibrium between the beta frequency of the human EEG and a GABA A receptor gene locus. Proc. Natl Acad Sci USA https://doi.org/10.1073/pnas.052716399 (2002).

29. Baumgarten, T. J. et al. Beta peak frequencies at rest correlate with endogenous GABA+/Cr concentrations in sensorimotor cortex areas. PLOS ONE https://doi.org/10.1371/journal.pone.0156829 (2016).

30. Rowland, L. M. et al. GABA predicts inhibition of frequency-specific oscillations in schizophrenia. J. Neuropsychiatry Clin. Neurosci. https://doi.org/10.1176/appi. neuropsych.11120368 (2013).

31. Yoon, J. H., Grandelis, A. \& Maddock, R. J. Dorsolateral prefrontal cortex GABA concentration in humans predicts working memory load processing capacity. J. Neurosci. https://doi.org/10.1523/JNEUROSCl.1970-16.2016 (2016).

32. Piers, T. M. et al. Translational concepts of mglur5 in synaptic diseases of the brain. Front. Pharmacol. https://doi.org/10.3389/fphar.2012.00199 (2012).

33. Kullmann, D. M. \& Lamsa, K. Roles of distinct glutamate receptors in induction of anti-Hebbian long-term potentiation. J. Physiol. 586, 1481-1486 (2008).

34. Newell, K. A. \& Matosin N. Rethinking metabotropic glutamate receptor 5 pathological findings in psychiatric disorders: Implications for the future of novel therapeutics. BMC Psychiatry https://doi.org/10.1186/1471-244X-14-23 (2014).

35. Holst, S. C. et al. Cerebral mGluR5 availability contributes to elevated sleep need and behavioral adjustment after sleep deprivation. Elife https://doi.org/ 10.7554/eLife.28751.001 (2017).

36. Lally, N. et al. Glutamatergic correlates of gamma-band oscillatory activity during cognition: a concurrent ER-MRS and EEG study. Neuroimage https://doi. org/10.1016/j.neuroimage.2013.07.049 (2014).

37. Bélanger, M., Allaman, I. \& Magistretti, P. J. Brain energy metabolism: focus on astrocyte-neuron metabolic cooperation. Cell Metab. https:/doi.org/10.1016/j. cmet.2011.08.016 (2011).

38. Tomasi, D., Wang, G.-J. \& Volkow, N. D. Energetic cost of brain functional connectivity. PNAS 110, 13642-13647 (2013).

39. Raichle, M. E. \& Mintun, M. A. Brain work and brain imaging. Annu. Rev. Neurosci. https://doi.org/10.1146/annurev.neuro.29.051605.112819 (2006).

40. Anticevic, A. et al. The role of default network deactivation in cognition and disease. Trends Cogn. Sci. https://doi.org/10.1016/j.tics.2012.10.008 (2012).

41. Menon, V. \& Uddin, L. Q. Saliency, switching, attention and control: a network model of insula function. Brain Struct Funct. 214, 655-667 (2010).

42. Attwell, D. \& Laughlin, S. B. An energy budget for signaling in the grey matter of the brain. J Cereb Blood Flow Metab. 21, 1133-1145. https://doi.org/10.1097/ 00004647-200110000-00001 (2001).
43. Sibson, N. R. et al. In vivo ${ }^{13} \mathrm{CNMR}$ measurements of cerebral glutamine synthesis as evidence for glutamate-glutamine cycling. Proc. Natl. Acad. Sci. 94, 2699-2704, https://doi.org/10.1073/pnas.94.6.2699 (1997).

44. Niswender, C. M. \& Conn, P. J. Metabotropic glutamate receptors: physiology, pharmacology, and disease. Annu. Rev. Pharm. Toxicol. 50, 295-322 (2010).

45. Sigel, E. \& Steinmann, M. E. Structure, function, and modulation of GABAA receptors. J. Biol. Chem. https://doi.org/10.1074/jbc.R112.386664 (2012).

46. Jacob, T. C., Moss, S. J. \& Jurd, R. GABAA receptor trafficking and its role in the dynamic modulation of neuronal inhibition. Nat. Rev. Neurosci. https://doi.org/ 10.1038/nrn2370 (2008).

47. Kim, Y. K., Yang, E. J., Cho, K., Lim, J. Y. \& Paik, N. J. Functional recovery after ischemic stroke is associated with reduced gabaergic inhibition in the cerebral cortex: a GABA PET study. Neurorehabil. Neural Repair https://doi.org/10.1177/ 1545968313520411 (2014).

48. Blaabjerg, L. \& Juhl, C. B. Hypoglycemia-Induced changes in the electroencephalogram: an overview. J. Diabetes Sci. Technol. https://doi.org/10.1177/ 1932296816659744 (2016)

49. Fukunaga, M. et al. Metabolic origin of BOLD signal fluctuations in the absence of stimuli. J. Cereb. Blood Flow Metab. https://doi.org/10.1038/jcbfm.2008.25 (2008).

50. Aiello, M. et al. Relationship between simultaneously acquired resting-state regional cerebral glucose metabolism and functional MRI: a PET/MR hybrid scanner study. Neuroimage 113, 111-121 (2015).

51. Levar, N., van Leeuwen, J. M. C., Denys, D. \& van Wingen, G. A. Divergent influences of anterior cingulate cortex GABA concentrations on the emotion circuitry. Neuroimage https://doi.org/10.1016/j.neuroimage.2017.06.055 (2017).

52. Northoff, G. et al. GABA concentrations in the human anterior cingulate cortex predict negative BOLD responses in fMRI. Nat. Neurosci. https://doi.org/ 10.1038/nn2001 (2007)

53. Arrubla, J., Tse, D. H. Y., Amkreutz, C., Neuner, I. \& Shah, N. J. GABA concentration in posterior cingulate cortex predicts putamen response during resting state fMRI. PLOS ONE 9, e106609 (2014).

54. Enzi, B. et al. Glutamate modulates resting state activity in the perigenual anterior cingulate cortex - a combined fMRI-MRS study. Neuroscience https:// doi.org/10.1016/j.neuroscience.2012.09.039 (2012).

55. Menon, V. Salience Network (Elsevier Inc., 2015).

56. Ametamey, S. M. et al. Radiosynthesis and preclinical evaluation of ${ }^{11} \mathrm{C}-\mathrm{ABP} 688$ as a probe for imaging the metabotropic glutamate receptor subtype 5 . J NuCl Med. 47, 698-705 (2006).

57. Pandey, G. N. et al. Benzodiazepine receptors in the post-mortem brain of suicide victims and schizophrenic subjects. Psychiatry Res. https://doi.org/ 10.1016/S0165-1781(97)00060-7 (1997).

58. Herzog, H. et al. High resolution BrainPET combined with simultaneous MRI. Nuklearmedizin 50, 74-82 (2011).

59. Rajkumar, R. et al. Simultaneous trimodal PET-MR-EEG imaging: do EEG caps generate artefacts in PET. PLOS ONE 1-16 (2017).

60. Sheehan, D. V. et al. The Mini-International Neuropsychiatric Interview (M.I. N.I.): the development and validation of a structured diagnostic psychiatric interview for DSM-IV and ICD-10. J Clin Psychiatry. 59 Suppl 20 22-57 (1998)

61. Harada, M., Kubo, H., Nose, A., Nishitani, H. \& Matsuda, T. Measurement of variation in the human cerebral GABA level by in vivo MEGA-editing proton MR spectroscopy using a clinical $3 \mathrm{~T}$ instrument and its dependence on brain region and the female menstrual cycle. Hum. Brain Mapp. https://doi.org/ 10.1002/hbm.21086 (2011).

62. Elmenhorst, D. et al. Circadian variation of metabotropic glutamate receptor 5 availability in the rat brain. J. Sleep. Res. 25, 754-761 (2016).

63. Canales-Candela, R., Riss, P. J. and Aigbirhio, F. I. Synthesis of $\left[{ }^{11} \mathrm{C}\right]$ Flumazenil ([ $\left.{ }^{11} \mathrm{C}\right] \mathrm{FMZ}$ ). In Radiochemical Syntheses (eds Scott, P. J. H. \& Hockley, B. G.). https://doi.org/10.1002/9781118140345.ch22 (2012).

64. Rota Kops, E., Hautzel, H., Herzog, H., Antoch, G. \& Shah, N. J. Comparison template-based versus $C T$-based attenuation correction for hybrid MR/PET scanners. IEEE Trans. Nucl. Sci. 62, 2115-2121 (2015).

65. Scheins, J. et al. Image-based Motion Correction for the Siemens hybrid-MR BrainPET Scanner. Nuklearmedizin 58, P107, https://doi.org/10.1055/s-00391683702 (2019)

66. Akkus, F. et al. Metabotropic glutamate receptor 5 neuroimaging in schizophrenia. Schizophr. Res. 183, 95-101 (2017).

67. Odano, I. et al. $\left[{ }^{18} \mathrm{~F}\right.$ Flumazenil binding to central benzodiazepine receptor studies by PET — quantitative analysis and comparisons with $\left[{ }^{11} \mathrm{C}\right]$ flumazenil. Neuroimage https://doi.org/10.1016/j.neuroimage.2008.12.005 (2009). 
68. Hammers, A. et al. Three-dimensional maximum probability atlas of the human brain, with particular reference to the temporal lobe. Hum. Brain Mapp. 19, 224-247 (2003).

69. Yan, C. G., Wang, X. D., Zuo, X. N. \& Zang, Y. F. DPABl: data processing \& analysis for (resting-state) brain imaging. Neuroinformatics 14, 339-351 (2016).

70. Bright, M. G. \& Murphy, K. Is fMRI 'noise' really noise? Resting state nuisance regressors remove variance with network structure. Neuroimage https://doi. org/10.1016/j.neuroimage.2015.03.070 (2015).

71. Friston, K. J., Williams, S., Howard, R., Frackowiak, R. S. J. \& Turner, R. Movementrelated effects in fMRI time-series. Magn. Reson. Med. 35, 346-355 (1996).

72. Stuart, A. In Rank Correlation Methods 2nd edn (ed. Kendall, M. G.). Br. J. Stat Psychol. 9 (1956).

73. Li, Z., Kadivar, A., Pluta, J., Dunlop, J. \& Wang, Z. Test-retest stability analysis of resting brain activity revealed by blood oxygen level-dependent functional MRI. J. Magn. Reson. Imaging 36, 344-354 (2012).

74. Menon, V. Large-scale brain networks and psychopathology: a unifying triple network model. Trends Cogn. Sci. 15, 483-506 (2011).

75. Delorme, A. \& Makeig, S. EEGLAB: an open source toolbox for analysis of single-trial EEG dynamics including independent component analysis. J. Neurosci. Methods 134, 9-21 (2004).

76. Oostenveld, R., Fries, P., Maris, E. \& Schoffelen, J. M. FieldTrip: Open source software for advanced analysis of MEG, EEG, and invasive electrophysiological data. Comput. Intell. Neurosci. https://doi.org/10.1155/2011/156869 (2011).

77. Niazy, R. K., Beckmann, C. F., lannetti, G. D., Brady, J. M. \& Smith, S. M. Removal of FMRI environment artifacts from EEG data using optimal basis sets. Neuroimage 28, 720-737 (2005).

78. Mullen, T. R. et al. Real-time neuroimaging and cognitive monitoring using wearable dry EEG. IEEE Trans. Biomed. Eng. https:/doi.org/10.1109/ TBME.2015.2481482 (2015).

79. Chang, C. Y., Hsu, S. H., Pion-Tonachini, L. \& Jung, T. P. Evaluation of artifact Subspace reconstruction for automatic artifact components removal in multichannel EEG recordings. IEEE Trans Biomed Eng. 67, 1114-1121. https://doi. org/10.1109/TBME.2019.2930186 (2020).

80. Marino, M. et al. Adaptive optimal basis set for BCG artifact removal in simultaneous EEG-fMRI. Sci. Rep. https://doi.org/10.1038/s41598-018-27187-6 (2018).

81. G. Gomez-Herrero et al. Automatic Removal of Ocular Artifacts in the EEG without an EOG Reference Channel, Proceedings of the 7th Nordic Signal Processing Symposium - NORSIG 2006, Rejkjavik, 2006, pp. 130-133, https://doi. org/10.1109/NORSIG.2006.275210.
82. Bell, A. J. \& Sejnowski, T. J. An information-maximization approach to blind separation and blind deconvolution. Neural Comput. https://doi.org/10.1162/ neco.1995.7.6.1129 (1995).

83. Winkler, I., Haufe, S. \& Tangermann, M. Automatic classification of artifactual ICA-components for artifact removal in EEG signals. Behav. Brain Funct. 7, 1-15. (2011).

84. Abreu, R. et al. Ballistocardiogram artifact correction taking into account physiological signal preservation in simultaneous EEG-fMRI. Neuroimage https://doi.org/10.1016/j.neuroimage.2016.03.034 (2016).

85. Lehmann, D. \& Skrandies, W. Reference-free identification of components of checkerboard-evoked multichannel potential fields. Electroencephalogr. Clin. Neurophysiol. 48, 609-621 (1980).

86. Tibshirani, R. \& Walther, G. Cluster validation by prediction strength. J. Comput. Graph. Stat. 14, 511-528 (2005).

87. Jurcak, V., Tsuzuki, D. \& Dan, I. 10/20, 10/10, and 10/5 systems revisited: their validity as relative head-surface-based positioning systems. Neuroimage $\mathbf{3 4}$, 1600-1611 (2007).

88. Fuchs, M., Kastner, J., Wagner, M., Hawes, S. \& Ebersole, J. S. A standardized boundary element method volume conductor model. Clin. Neurophysiol. https://doi.org/10.1016/\$1388-2457(02)00030-5 (2002).

89. Mazziotta, J. et al. A probabilistic atlas and reference system for the human brain: International Consortium for Brain Mapping (ICBM). Philos. Trans. R. Soc. B https://doi.org/10.1098/rstb.2001.0915 (2001).

90. Shirer, W. R., Ryali, S., Rykhlevskaia, E., Menon, V. \& Greicius, M. D. Decoding subject-driven cognitive states with whole-brain connectivity patterns. Cereb. Cortex 22, 158-165 (2012).

91. Custo, A., Vulliemoz, S., Grouiller, F., Van De Ville, D. \& Michel C. EEG source imaging of brain states using spatiotemporal regression. Neuroimage https:// doi.org/10.1016/j.neuroimage.2014.04.002 (2014).

92. Benjamini, Y. \& Hochberg, Y. Controlling the false discovery rate: a practical and powerful approach to multiple testing. J. R. Stat. Soc. Ser. B https://doi.org/ 10.1111/j.2517-6161.1995.tb02031.x (1995).

93. Dunn O. J. Multiple comparisons among means. J. Am. Stat. Assoc. https://doi. org/10.1080/01621459.1961.10482090 (1961).

94. Cardillo, G. (2020). Dunn's test (https://github.com/dnafinder/dunn), GitHub. Retrieved December 9, 2019.

95. Groppe, D. M., Urbach, T. P. \& Kutas, M. Mass univariate analysis of eventrelated brain potentials/fields I: a critical tutorial review. Psychophysiology $4 \mathbf{8}$, 1711-1725 (2011) 